\title{
Multiple steady states on an adsorbent surface
}

\author{
A. Córdoba and J. J. Luque \\ Departamento de Termologí, Facultad de Física, Universidad de Sevilla, Sevilla, Spain
}

(Received 22 July 1985)

\begin{abstract}
The occurrence of multiple steady states in a system formed by monomers adsorbed on a lattice, with attractive interaction between nearest adatoms, is demonstrated. This fact causes hysteresis in the adsorption-desorption or desorption-readsorption processes. On the other hand, the coordination number of the adsorbent lattice and the corresponding degree of nonlinearity of the rate equation do not essentially influence the results obtained.
\end{abstract}

\section{INTRODUCTION}

Interactions between adatoms have helped explain adsorption energies which are functions of coverage. These interactions influence the rate of surface reactions, making them quicker or slower, thus producing "autocatalytic" mechanisms. As is well known, multiple steady states can exist in chemical reactions including autocatalytic steps. ${ }^{1}$ A steady state may be stable or unstable according to whether a small deviation from it decays to the original steady state or evolves away from it.

In several previous papers ${ }^{2-4}$ we have dealt with the kinetics of various adsorption processes from an extension of the Ising-Glauber model $^{5}$ where, starting from a master equation and suitably chosen transition probabilities, the macroscopic equations governing the time evolution of the system were obtained. When interactions between adatoms exist, the kinetic equations involve nonlinear terms corresponding to these interactions. As shall be demonstrated, multiple steady states, for a range of the parameters characterizing the problem, can exist. Several of these steady states would be stable. Thus, initial conditions essentially determine the final steady state reached by the system and hysteresis phenomena can take place. The simplest case is the adsorption-desorption of a monomer, with a constant interaction energy existing only between nearest neighbors.

\section{THE MODEL}

We consider a particle which can be adsorbed on a vacant site of a linear chain (later we shall extend the results to the case of a lattice with coordination number $z$ ). Conversely, an adsorbed particle can be desorbed, thus creating a vacant site. Schematically, the process may be represented as

$$
A(v)+V(s) \rightleftarrows X(s)
$$

where $A, X$, and $V$ denote, respectively, the particles of the vapor, the adatoms, and the vacancies on the adsorbent lattice.

When interactions between adatoms exist, the above simple scheme is not valid, because the interactions favor or retard the adsorption-desorption mechanism, and in general more than one particle is involved in each elemental process. Thus, this reaction is autocatalytic and the rate equations are nonlinear. This fact suggests the existence of multiple steady states.
We assume that the process takes place as follows: The transition probability of each elemental process is chosen in the Arrhenius form, and the activation energy is decomposed into two additive terms, corresponding to the action of the substrate and to the interaction between nearest adatoms, respectively. If the plus sign denotes a filled site and the minus sign a vacant one, the elemental processes taking place on the lattice and their respective probabilities are as follows. Adsorption:

$$
\begin{array}{ll}
+-+\rightarrow+++, & W_{++}=A \exp \left[-\left(E+2 E_{i}\right) / k T\right], \\
+--\rightarrow++-, & W_{+-}=A \exp \left[-\left(E+E_{i}\right) / k T\right], \\
--+\rightarrow-++, & W_{-+}=A \exp \left[-\left(E+E_{i}\right) / k T\right], \\
---\rightarrow-+-, & W_{--}=A \exp (-E / k T) .
\end{array}
$$

Desorption:

$$
\begin{array}{ll}
+++\rightarrow+-+, & W_{++}^{\prime}=A^{\prime} \exp \left[-\left(E^{\prime}+2 E_{i}^{\prime}\right) / k T\right], \\
++-\rightarrow+--, & W_{+-}^{\prime}=A^{\prime} \exp \left[-\left(E^{\prime}+E_{i}^{\prime}\right) / k T\right], \\
-++\rightarrow--+, & W_{-+}^{\prime}=A^{\prime} \exp \left[-\left(E^{\prime}+E_{i}^{\prime}\right) / k T\right], \\
-+-\rightarrow---, & W_{--}^{\prime}=A^{\prime} \exp \left(-E^{\prime} / k T\right) .
\end{array}
$$

Here, $A$ and $A^{\prime}$ are constant factors, $E$ and $E^{\prime}$ are the contribution of the substrate to the total activation energy, $E_{i}$ and $E_{i}^{\prime}$ are the contribution to the total activation energy due to interaction between two neighbor adatoms, $k$ is the Boltzmann constant, and $T$ is the temperature. An attractive interaction facilitates adsorption and makes desorption difficult, and therefore $E_{i}<0$ and $E_{i}^{\prime}>0$. On the contrary, a repulsive interaction facilitates desorption and makes adsorption difficult, and therefore $E_{i}>0$ and $E_{i}{ }^{\prime}<0$. Thus $E_{i}$ and $E_{i}{ }^{\prime}$ have different signs. For the sake of simplicity, we have assumed that all the preexponential factors are the same for the different cases ( $A$ for adsorption and $A^{\prime}$ for desorption).

Starting from this model and following the guideline of Ref. 2, we obtain a hierarchy of rate equations. The first equation of this hierarchy is

$$
\begin{aligned}
\frac{d n_{+}}{d t}= & n_{+-+} W_{++}+2 n_{+--} W_{+-}+n_{---} W_{--} \\
& -n_{+++} W_{++}^{\prime}-2 n_{++-} W_{+-}^{\prime}-n_{-+-} W_{--}^{\prime},
\end{aligned}
$$

where $n_{i}$ and $n_{i j k}(i, j, k \rightarrow+,-)$ denote the fractions of singlets and triplets in $i$ and $i-j-k$ states, respectively. 
In order to close this hierarchy at a finite level, it is necessary to use some approximation method. We have chosen the Bragg-Williams approximation, so that $n_{i j k}$ $=n_{i} n_{j} n_{k}$, and we retain only the first equation of the hierarchy. Introducing this approximation into Eq. (2), we obtain

$$
\frac{d \Theta}{d(H t)}=(1-\Theta)(J \Theta+1)^{2}-h \Theta\left(J^{\prime} \Theta+1\right)^{2}=P_{3}(\Theta),
$$

where $\Theta=n_{+}$denotes the coverage, $J=\exp \left(-E_{i} / k T\right)-1$, $J^{\prime}=\exp \left(-E_{i}^{\prime} / k T\right)-1, H=A \exp (-E / k T)$, and $h=\left(A^{\prime} /\right.$ $A) \exp \left[-\left(E^{\prime}-E\right) / k T\right]$.

\section{RESULTS AND DISCUSSION}

To calculate the steady values of $\Theta, \Theta_{s}$, we consider $d \Theta / d(H t)=P_{3}(\Theta)=0$, which is an algebraic equation of the third degree, and therefore has three roots. It can easily be demonstrated that there is always one real root in the range $0<\Theta<1$, and it is also possible that three roots exist in the above range. In this case, two roots correspond to stable states, both of which are separated by an unstable one.

The number of steady states depends on the values of the parameters $J, J^{\prime}$, and $h$. We assume $\left|E^{\prime}\right|=|E|$ and, therefore, $J^{\prime}=-J /(J+1)$. Thus the behavior of the system is determined by only two parameters, $J$ and $h$.

The stability diagram in the $J-h$ plane is shown in Fig. 1, where 1 and 3 denote the number of steady states (for case 3 , only two steady states are stable). The $a$ and $b$ lines, separating the different regions of the diagram, coincide at a "critical" point, $C$, which is defined by $h_{C}=9$ and $J_{C}=2$. For $J<J_{C}$ or $h<h_{C}$ there is only one steady state. We can conclude that, for a repulsive or a slightly attractive lateral interaction such that $E_{i}>-k T \ln 3$, multiplicity of steady states does not exist. This multiplicity is possible only for $E_{i}<-k T \ln 3$ and a value of $h$ in the suitable range

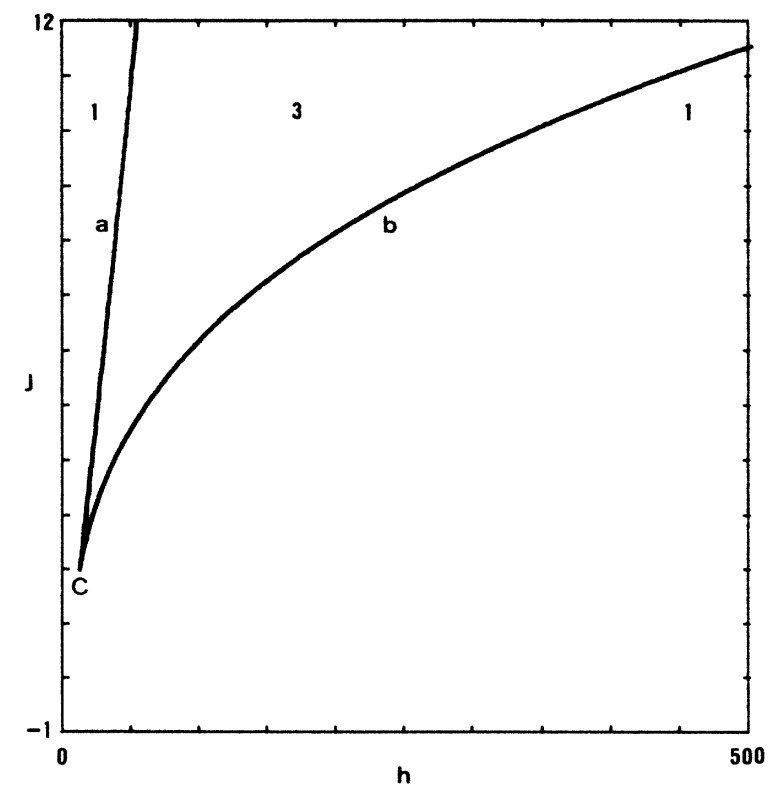

FIG. 1. Stability diagram in the $(J, h)$ plane. 1 and 3 denote the number of steady states in each region of the plane. (between the $a$ and $b$ lines). As $J$ increases, the range of $h$ giving multiple steady states becomes larger and larger. The value of $E_{i}$ necessary to cause multiplicity of steady states is quite reasonable. Thus, at room temperature, $\left|E_{i}\right| \simeq 1$ $\mathrm{kcal} / \mathrm{mol}$ is enough for this phenomenon to take place, if a suitable value is given to $h$; for two-dimensional lattices, as we shall see later, and for higher temperatures, this value of $E_{i}$ is still smaller.

In Fig. 2, $\Theta_{s}$ vs $h$ for different values of $J$ is plotted. One can observe the similarity between these curves and the isotherms of a van der Waals gas and, therefore, the analogy between this phenomenon and first-order phase transitions. ${ }^{6}$

An interesting parameter is the relaxation time (scaled by $H$ ) of the degree of adsorption far from a stable state. We have found that essentially two relaxation times exist, corresponding to initial and final stages, respectively. The final relaxation time, $\tau$, does not depend on the initial state but does characterize the steady state. The behavior of $\tau$ depends on $J$ and $h$, and is shown in Fig. 3. For values of $J$ less than $J_{C}$ there is obviously only one relaxation time, which increases as $h$ decreases (and, consequently, $\Theta_{s}$ increases). As regards values of $J$ greater than $J_{C}$, for $h<h_{a}$ ( $h_{a}$ denoting the value of $h$ corresponding to critical $a$ line at a given $J$ ), there is only one relaxation time, $\tau_{1}$, which increases smoothly as $h$ increases. When $h=h_{a}$, while $\tau_{1}$ follows the aforementioned behavior, a relaxation time $\tau_{2}$, corresponding to a new steady state, emerges. At the beginning, $\tau_{2}$ is very great $\left(\tau_{2} \rightarrow \infty\right.$ as $\left.h \rightarrow h_{a}\right)$, decreases abruptly, and then continues decreasing smoothly, even at $h=h_{b}$ ( $h_{b}$ denoting the value of $h$ corresponding to critical $b$ line at a given $J)$. At $h=h_{b}, \tau_{1} \rightarrow \infty$, and for $h>h_{b}$ only $\tau_{2}$ exists. As $J$ decreases, the difference $h_{b}-h_{a}$ decreases and at the critical point, where $h_{b}=h_{a}$, both steady states coalesce and the relaxation time tends to become infinite.

Finally, we extend the formulation of this problem to a two-dimensional lattice with coordination number $z(z$ $=3,4,6$ ). Again, we follow the guideline of Ref. 2, extended to a two-dimensional case, and use the Bragg-Williams

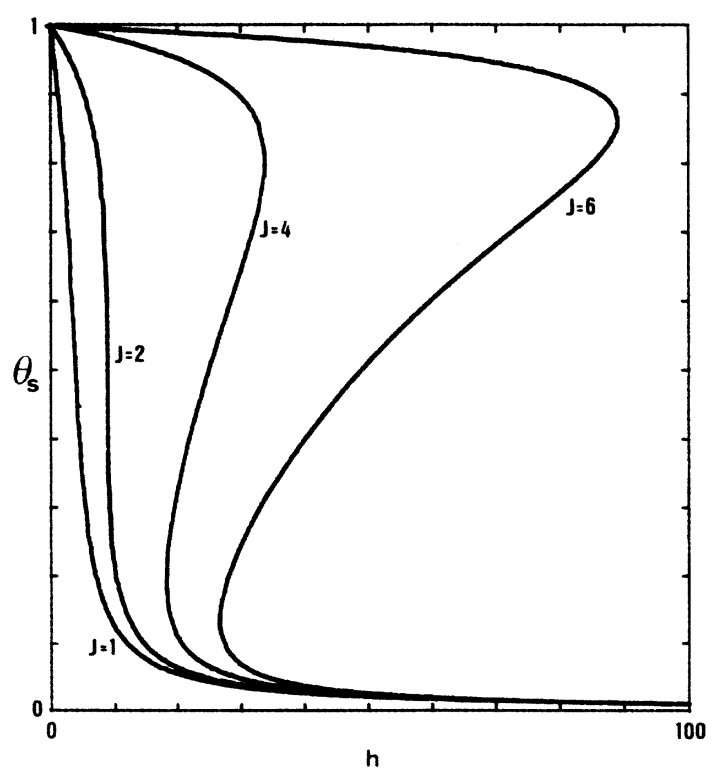

FIG. 2. Steady states of the system as a function of $h, J$ being a parameter. 


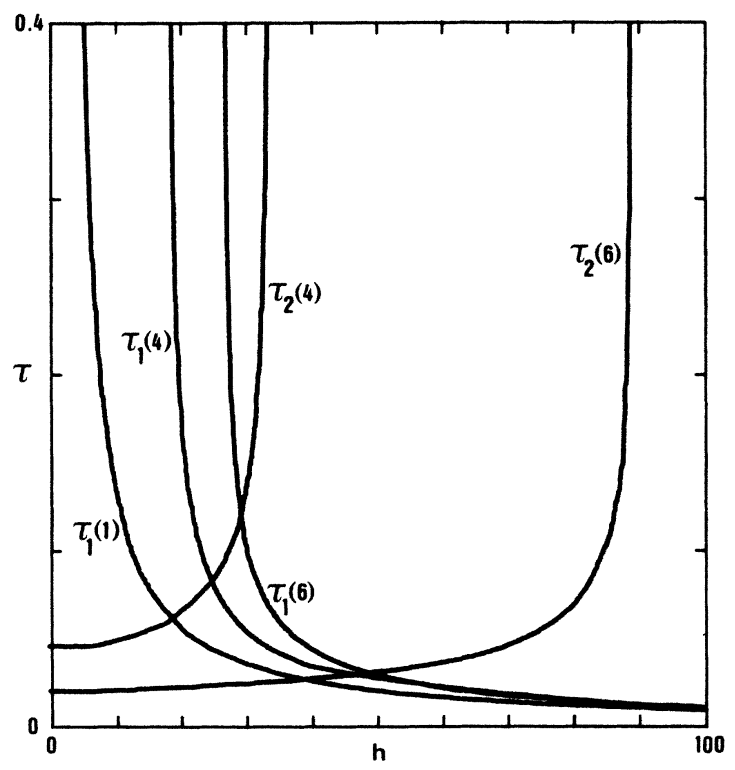

FIG. 3. Scaled relaxation time as a function of $h, J$ being a parameter. The numbers in brackets denote the values of $J$.

approximation. We then obtain the kinetic equation

$\frac{d \Theta}{d(H t)}=(1-\Theta)(J \Theta+1)^{z}-h \Theta\left(J^{\prime} \Theta+1\right)^{z}=P_{z+1}(\Theta)$.

The right-hand side of (3) is a polynomial of the $(z+1)$ th degree and we must solve the equation $P_{z+1}(\Theta)=0$ to find the steady states. One should expect that the stability diagram shown in Fig. 1 drastically changes, because the equation $P_{z+1}(\Theta)=0$ has, in general, $z+1$ roots. However, this change does not take place. We have performed numerical calculations for $J$ going from -1 to very high values, and for $h$ from very low positive values to very high ones, i.e.,

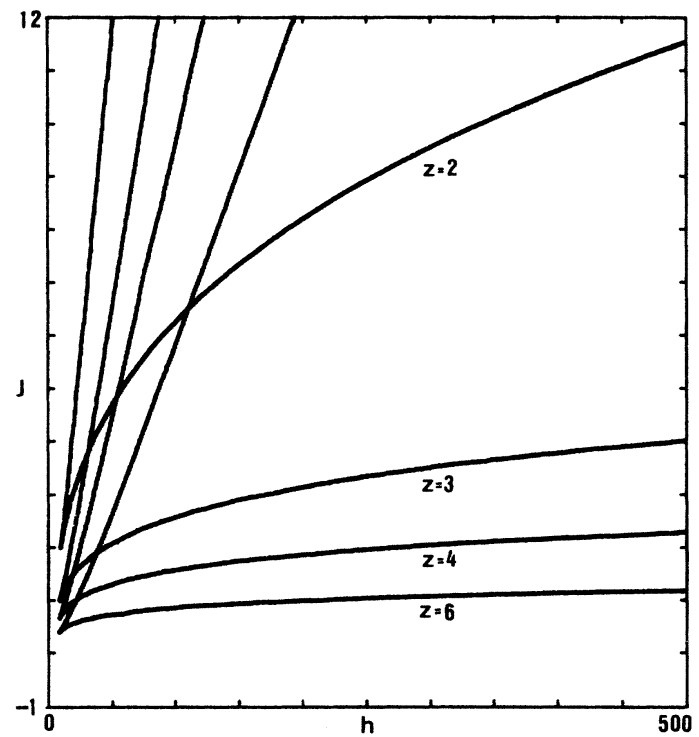

FIG. 4. Stability diagram in the $(J, h)$ plane for various twodimensional lattices with coordination number $z$ and comparison with the linear chain.

we have swept practically all the region of the plane $J>-1, h>0$. We have always found one or three steady states (two of the states being stable in the latter case) and the stability diagrams in the $J-h$ plane are similar to that obtained for the linear chain, with critical points $J_{C}=1.000$ and $h_{C}=8.000$, for $z=3 ; J_{C}=0.666$ and $h_{C}=7.716$, for $z=4$; and $J_{C}=0.400$ and $h_{C}=7.529$, for $z=6$ (Fig. 4). Thus, we can conclude that, for the two-dimensional case, the lattice coordination number and the degree of nonlinearity of the rate equation, which are correspondingly greater than those of the one-dimensional case, do not essentially influence the results of the problem.
${ }^{1} \mathrm{G}$. Nicolis and I. Prigogine, Self-Organization in Nonequilibrium Systems (Wiley-Interscience, New York, 1977).

2J. J. Luque and A. Córdoba, J. Chem. Phys. 76, 6393 (1982).

${ }^{3}$ A. Córdoba and J. J. Luque, Phys. Rev. B 26, 4028 (1982); 28, 7353 (1983).
${ }^{4}$ A. Córdoba and J. J. Luque, Phys. Rev. B 31, 8111 (1985).

${ }^{5}$ R. J. Glauber, J. Math. Phys. 4, 294 (1963).

${ }^{6}$ A. Nitzan, P. Ortoleva, J. Deutch, and J. Ross, J. Chem. Phys. 61, 1056 (1974). 